\title{
Phase Sensitive Reconstruction for Water/Fat Separation in MR Imaging Using Inverse Gradient
}

\author{
Joakim Rydell $^{1,2}$, Hans Knutsson ${ }^{1,2}$, Johanna Pettersson ${ }^{1,2}$, \\ Andreas Johansson ${ }^{2}$, Gunnar Farnebäck ${ }^{2}$, Olof Dahlqvist ${ }^{1,3}$, \\ Peter Lundberg $^{1,3}$, Fredrik Nyström ${ }^{4}$, and Magnus Borga ${ }^{1,2}$ \\ ${ }^{1}$ Center for Medical Image Science and Visualization (CMIV) \\ ${ }^{2}$ Department of Biomedical Engineering, Linköping University, Sweden \\ ${ }^{3}$ Department of Medicine and Care, Linköping University, Sweden \\ ${ }^{4}$ Department of Endocrinology and Metabolism, Linköping University, Sweden
}

\begin{abstract}
This paper presents a novel method for phase unwrapping for phase sensitive reconstruction in MR imaging. The unwrapped phase is obtained by integrating the phase gradient by solving a Poisson equation. An efficient solver, which has been made publicly available, is used to solve the equation. The proposed method is demonstrated on a fat quantification MRI task that is a part of a prospective study of fat accumulation. The method is compared to a phase unwrapping method based on region growing. Results indicate that the proposed method provides more robust unwrapping. Unlike region growing methods, the proposed method is also straight-forward to implement in 3D.
\end{abstract}

\section{Introduction}

In MRI, reconstructed images are in general complex valued, but usually only the magnitude of the signal is saved and the resulting image is therefore real valued and positive. In many MRI applications, however, image analysis based on complex valued data dramatically improves the ability to acquire accurate estimates of physiological parameters. In some of these applications the analysis may be improved if an unwrapped phase field of the complex images is also accurately determined. The actual phase of the MRI signal may depend on hardware and experimental parameters such as the main B0 field homogeneity, echo time (TE), receiver and excitation coil sensitivity, but also on the choice of pulse sequence and tissue dependent factors.

One particular example were phase unwrapping can be very useful is in quantitative imaging of fat, something that is useful in obesity studies. In this paper, we present a novel phase unwrapping method and show how it can be used for quantitative fat imaging. The method is compared to an established method [1] on images obtained in a fat accumulation study.

There is a tremendous increase in the prevalence in obesity worldwide. It is well known that obesity, in particular the male abdominal fat accumulation 
(apple shape) pattern is associated with high risk for development of type 2 diabetes, high blood pressure, and disturbed cholesterol levels. It is generally considered that the unfavorable prognosis in sedentary subjects with abdominal obesity is due to large amounts of intra abdominal fat [2]. However, this theory has not earlier been tested in prospective studies. The work presented here is a part of such an ongoing study of the impact on intra abdominal fat accumulation by fast food based weight gain combined with reduced physical activity.

\section{Background}

Differentiating tissues that mainly contain water from fat tissues in the abdomen is often performed using heavily T1 weighted images as these provide high contrast between fat tissues with short T1 and surrounding tissue [2 3]. Unfortunately this approach is very sensitive to partial volume effects as the tissue types are often not well separated. Donnelly et al evaluated segmentation of imaging data acquired using several different techniques. Results from a phantom modeling abdominal water and fat content showed that the fat volume fraction of the tissue is consequently underestimated using fat segmentation of T1 weighted images [4. A better solution is to image the water and fat content both separately and together, by the application of constant level appearance (CLEAR) image reconstruction. This method removes the effect of inhomogeneous sensitivity profiles of the acquisition coils. Using this technique the estimated fat content is virtually independent on voxel size as well as partial volume effects as no classification of the voxels into water/fat is needed.

The two-point Dixon technique [5] enables the separation of water and fat signals in each individual voxel by signal acquisition using two different TEs in a gradient echo imaging sequence. At $\mathrm{TE}_{1}$ the water and fat signal are measured $180^{\circ}$ out of phase, and at $\mathrm{TE}_{2}$ they are detected in phase. Without any other phase variations than those caused by the different resonance frequencies of water and fat, both the in phase and out of phase images would be real valued. In the image acquired at $\mathrm{TE}_{2}$, where water and fat are in phase, both components would contribute to positive signal values, i.e. $I_{2}=w+f$. In the out of phase image, $I_{1}$, water would contribute with positive values while fat contributes with negative values, i.e. $I_{1}=w-f$. Then the water component could be obtained as $\left(I_{1}+I_{2}\right) / 2$ and the fat could be obtained as $\left(I_{2}-I_{1}\right) / 2$. However, due to experimental factors, the complex phase varies across the images. Because of the spatially varying phase offset, both images are complex:

$$
\begin{aligned}
& I_{1}=(w-f) e^{i \phi_{1}} \\
& I_{2}=(w+f) e^{i \phi_{2}}
\end{aligned}
$$

where $\phi_{1}$ and $\phi_{2}$ are spatially varying phase fields. Hence the images need phase correction before the water and fat images can be calculated. In the in phase image, $I_{2}$, correcting the phase variations is easy. Since the water and fat components $(w$ and $f$ ) are both positive, $w+f$ is also always positive and the corrected 
image $\tilde{I}_{2}$ is simply obtained as the magnitude of $I_{2} ; \tilde{I}_{2}=\left\|I_{2}\right\|=w+f$. However, since the sign of $w-f$ is unknown, the out of phase image $I_{1}$ can not be corrected in the same way. Instead an estimate of the phase field $\phi_{1}$ is needed. When that field is known, a corrected image $\tilde{I}_{1}$ can be calculated as

$$
\tilde{I}_{1}=I_{1} e^{-i \tilde{\phi}_{1}} \approx w-f
$$

where $\tilde{\phi}_{1}$ is the estimate of the true phase field $\phi_{1}$.

$\tilde{\phi}_{1}$ can be estimated in several different ways, a few of which are presented in $6 / 7 / 8$. In section 3.3 a method by Ma [1] is described along with the proposed method for calculating $\tilde{\phi}_{1}$.

\section{Materials and Methods}

\subsection{Material}

After ethical approval, we recruited 12 healthy lean males and 6 females as volunteers. The participants had to be willing to accept an increase in body weight of $5-15 \%$ and to eat at least two fast food-based meals a day, preferably at well known fast food restaurants such as McDonald's and Burger King, for four weeks. All subjects continually had contact with dieticians during the study. The dietary advice was individually adjusted to result in an intake corresponding to doubling the caloric need. Physical activity was not to exceed 5000 steps per day. If a study subject reached a weight-gain of $15 \%$ he or she terminated the study as soon as possible. The subjects were $26 \pm 6.6$ years old and the mean increase in body weight was $10 \%$. The corresponding change in body-mass index was from $21.9 \pm 1.9$ to $23.9 \pm 2.2 \mathrm{~kg} / \mathrm{m}^{2}$. Five of the 18 subjects reached the maximal $15 \%$ increase in body weight.

\subsection{Data Acquisition}

Images of the 18 subjects were acquired before and after the study, using a 1.5 Tesla Philips Achieva MR-scanner (R2). A four element sensitivity encoding (SENSE) body coil was positioned to provide as high signal to noise ratio (SNR) as possible from the level of the diaphragm to the bottom of the pelvis. No SENSE acceleration was used, i.e. the SENSE coil was only used to obtain a high SNR. Magnitude and phase images were acquired separately from two different stacks using a field of view (FOV) of $290 \times 410 \mathrm{~mm}, 5 \mathrm{~mm}$ slice thickness, 40 slices and $1.6 \times 1.6 \mathrm{~mm}$ in-plane resolution. The images were obtained at two different TEs using a multi-slice spoiled fast gradient echo pulse sequence. The first acquisition was obtained using $\mathrm{TE}_{1}=2.3 \mathrm{~ms}$ with the fat and water signals $180^{\circ}$ out of phase, and the second using a $\mathrm{TE}_{2}=4.6 \mathrm{~ms}$ with the fat and water in phase. The repetition time (TR) was $286 \mathrm{~ms}$. Data was collected using breathhold technique. CLEAR reconstruction, which removes the effect of acquisition coil inhomogeneities, was used. 


\subsection{Data Processing}

Two methods for phase unwrapping have been implemented: a method based on region growing by Ma [1] and the proposed method. The method by Ma has been implemented for comparison with the proposed technique and is presented in more detail in the following section. The section after that presents the proposed method. Each of these methods provides an estimate $\tilde{\phi}_{1}$ of the phase field, which is used to calculate a corrected out of phase image $\tilde{I}_{1}$ according to equation 3 . It should be noted that neither of the methods is limited to water/fat separation. These methods solve the more general phase unwrapping problem, and are useful also for other applications where phase sensitive reconstruction is needed.

Region Growing Based Phase Unwrapping. A number of methods for phase correction of images acquired using a two-point or three-point Dixon technique are based on tracking the phase evolution with a region growing algorithm 617. The method presented by Ma in [1 uses both phase and amplitude information to make the region growing process more robust. It performs phase correction by traversing the image pixel by pixel and for each pixel determines whether it mostly contains water or fat. Two phase-gradient maps, $G_{x}$ and $G_{y}$, representing the phase difference in the $x$ and $y$ directions respectively, in combination with multiple pixel stacks, are used to determine the order in which the pixels are traversed. The idea is to choose the order according to the amount of phase variation in the image. Pixels with lower variation should be visited before pixels with higher variations to obtain a more robust processing.

To initiate the algorithm nine empty pixel stacks, one for each $10^{\circ}$ interval from $0^{\circ}$ to $90^{\circ}$, are created. The phase-gradient maps are computed as the phase difference between two neighboring pixels along the $x$ and $y$ axes, respectively. An arbitrary pixel in the image is chosen as initial seed and put onto a pixel stack to start region growing. The following three steps are then repeated until all pixels have been checked:

1. Select the seed from the lowest non empty pixel stack.

2. Visit the four nearest neighbors, if not already visited, and place them onto the pixel stacks according to their $G_{x}$ or $G_{y}$ value, depending on from which direction the pixel is visited. If the value is in the $0^{\circ}-10^{\circ}$ interval the pixel is placed on the first pixel stack, if the value is in the $10^{\circ}-20^{\circ}$ interval it is placed on the second stack, etc.

3. Finally the phase value of the seed pixel is determined based on the neighboring pixels that have already been checked. If the phase difference between the seed pixel and a summation of already visited pixels within a defined boxcar region exceeds $90^{\circ}$ the sign of the seed pixel is flipped.

When the current seed pixel has been checked a new one is chosen as described in step one. The result of the region growing process is a complex image without the phase discontinuities caused by the fat-water boundaries. Therefore the phase in each pixel of the resulting image describes the phase due to the local field inhomogeneity. 
Inverse Gradient Method. We propose the following estimation procedure:

1. Calculate a synthetic in phase image $I_{1}^{*}$ as

$$
I_{1}^{*}=\left\|I_{1}\right\| e^{i 2 \arg \left(I_{1}\right)}
$$

In $I_{1}^{*}$ the phase at each pixel will be twice the phase in $I_{1}$, which means that signals from water and fat will be in phase. The phase error due to magnetic field inhomogeneities will be twice as large as $\phi_{1}$.

2. Find the gradient of the phase of $I_{1}^{*}$, i.e. a vector field describing how the phase of $I_{1}^{*}$ changes with a small step in the $x$ or $y$ direction.

3. Divide the gradient field by 2 . This is needed since the gradient is estimated from $I_{1}^{*}$, where the phase varies twice as fast as in $I_{1}$.

4. Use normalized convolution 9] to interpolate the gradient field in regions where the phase estimates can be expected to be very noisy.

5. Integrate the gradient field to obtain $\tilde{\phi}_{1}$, the estimate of the phase error $\phi_{1}$. The integration procedure is described below. The integration problem is only solved in the image region corresponding to the body of the patient. Thus random phase variations outside the interesting region do not affect the resulting phase estimate.

At a first glance it may not be obvious that differentiating the phase and then integrating it again will have any effect on the final result. The rationale behind this operation is that while the phase itself contains discontinuities where it wraps from $-\pi$ to $\pi$, the gradient field is smooth. Integrating the gradient field yields an unwrapped phase which is not constrained to the interval $[-\pi, \pi]$. Hence the unwrapped phase can be divided by two without the ambiguities which arise when the number of multiples of $2 \pi$ is unknown.

The reason for using the synthetic in phase image $I_{1}^{*}$ with more rapid phase variation instead of the original out of phase image is that $I_{1}$ has phase discontinuities at the boundaries between water and fat. Such discontinuities adversely affect the estimate of the phase gradient. When the in phase image is used for estimating the phase, no discontinuities between different tissue types exist. It might be argued that the actual in phase image $I_{2}$ should be used instead of the synthetic image $I_{1}^{*}$. However, the phase variation in $I_{2}$ is not always exactly twice as fast as that in $I_{1}$ and thus the synthetic image $I_{1}^{*}$ is needed for correcting $I_{1}$.

In regions where the signal intensity is very low, the phase of $I_{1}^{*}$ is uncertain. Such regions include air filled cavities in the body and regions near boundaries between fat and water. In $I_{1}$ regions near such boundaries have low signal strength since the out of phase signals from water and fat cancel. Hence $I_{1}^{*}$, which is derived from $I_{1}$, also suffers from signal loss in those regions. Normalized convolution is used to interpolate the phase gradient in uncertain regions.

Integration of the phase gradient field $\mathbf{g}$ over a bounded and connected domain $\Omega$ is done by finding the scalar function $\phi$ whose gradients in a least squares sense are closest to $\mathrm{g}$ over $\Omega$. It can be shown by calculus of variations 810 that $\phi$ satisfies a Poisson equation with inhomogeneous Neumann boundary conditions, 


$$
\begin{cases}\Delta \phi(\mathbf{x})=\nabla \cdot \mathbf{g}(\mathbf{x}), & \text { all } \mathbf{x} \in \Omega \\ \frac{\partial \phi}{\partial n}(\mathbf{x})=\mathbf{n} \cdot \mathbf{g}(\mathbf{x}), & \text { all } \mathbf{x} \in \partial \Omega\end{cases}
$$

This partial differential equation needs to be solved numerically. There are offthe-shelf solvers available [11] but usually they are limited to rectangular domains $\Omega$ and sometimes also to simpler boundary conditions than the inhomogeneous Neumann. The limitation to rectangular domains can be worked around by solving the problem on a rectangular bounding box and iteratively updating the gradients outside $\Omega$ but this comes at a computational cost and can under some circumstances introduce systematic errors in the solution. Instead we have developed our own very efficient solver based on the full multi-grid method [12] which works directly on the specified domain. We have implemented the solver in $2 \mathrm{D}$ and $3 \mathrm{D}$ as a $\mathrm{C}$ code mex file for Matlab, and made it available for free download.

Because of the interpolation and the noisy phase estimates, the gradient field obtained after step 4 above is not guaranteed to be conservative. This means that the phase difference between two points in the image may be different depending on the choice of path between the points. Hence the integrated phase correction field is not perfect. To overcome this problem the algorithm is iterated approximately 10 times. Figures 1 a and 2 a show two examples of uncorrected images $I_{1}$. Corrected images $\tilde{I}_{1}$ are shown in figures $1 \mathrm{f}$ and $2 \mathrm{f}$. In figures $1 \mathrm{f}$ and 22 intermediate results after one iteration are shown.

As can be seen in figures 1f and 2f, there is almost no phase variation within water or fat after the correction. However, the phase angles of water and fat are not necessarily $0^{\circ}$ and $180^{\circ}$, respectively, but instead any two complex angles $180^{\circ}$ apart. Finding these angles and compensating for the constant phase offset is easy and yields a real valued image. One problem remains, though: there may still be a phase offset of $180^{\circ}$, i.e. fat and water may be interchanged. However, it is known that the strongest signal originates from fat. Hence the sign of the corrected image is flipped if the signal with the largest magnitude is positive. After this final correction, we obtain $\tilde{I}_{1}=w-f$ and the pure water and fat images can be calculated according to the equations in section 2 .

The proposed method is related to the method presented by Song et al in [8], but that method is based on a three-point Dixon acquisition, i.e. it uses a third image, acquired at another echo time, to estimate the phase correction. In contrast, the proposed method estimates the phase correction using only the out of phase image $I_{1}$ and a synthetic in phase image derived from this image. This is necessary in order to be able to process these data sets since the phase error $\phi_{1}$ can not be derived from the in phase image $I_{2}$ (see above). Another difference is that the method by Song solves the Poisson equation in a sequence of regions with consistent phase, while the proposed method solves the equation directly using a fast multi-grid solver.

$\overline{{ }^{1} \text { http://www } . i s y . l i u . s e / ~} \sim$ gf/software/ 


\section{Results}

Figure 1 demonstrates the result for a slice from one data set. The uncorrected out of phase image is shown along with images corrected by phase estimates from the method based on region growing and from the proposed method. Fat and water images calculated according to the equations in section 2 , using the corrected images $\tilde{I}_{1}$ and $\tilde{I}_{2}$, are also shown. The differences in the result for the two methods are minimal for this slice.

The slice in figure 2 is a more difficult case. The amount of phase wrap is much larger for this slice. The differences between the two methods are still not very large, but can be seen in some regions. A close-up of one of the regions in figure 2 where the differences are quite obvious is shown in figure 3. The first thing one notices is that the region belonging to the patient's arm has been classified differently by the two methods. This is, however, not relevant for measuring the abdominal fat. A more relevant difference is that a part of the subcutaneous fat, next to the arm, has obviously been misclassified by the

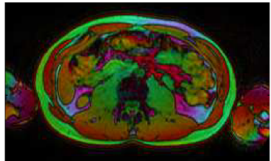

(a) Out of phase image

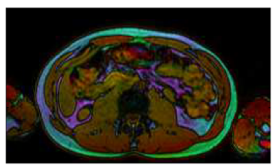

(e) After 1 iteration

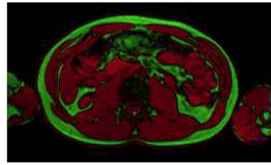

(b) Corrected image (Ma method)

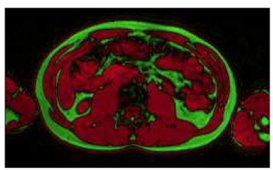

(f) Corrected image (our method)

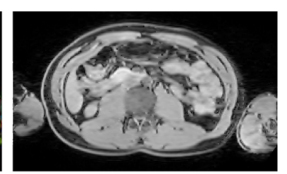

(c) Water image (Ma method)

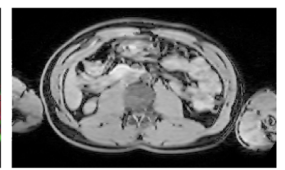

(g) Water image (our method)

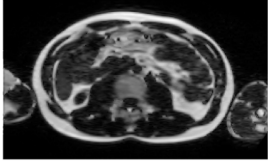

(d) Fat image (Ma method)

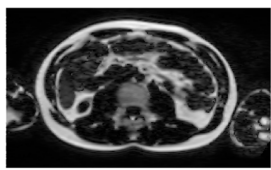

(h) Fat image (our method)

Fig. 1. Images from data set 1

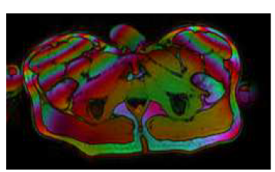

(a) Out of phase image

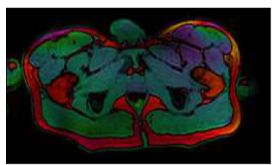

(e) After 1 iteration

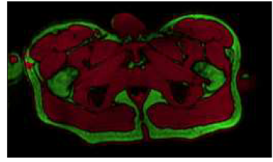

(b) Corrected image (Ma method)

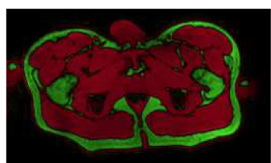

(f) Corrected image (our method)

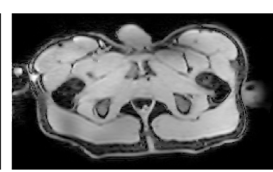

(c) Water image (Ma method)

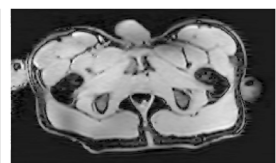

(g) Water image (our method)

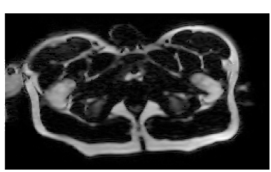

(d) Fat image (Ma method)

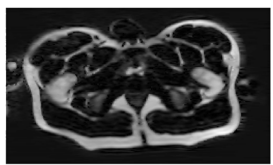

(h) Fat image (our method)

Fig. 2. Images from data set 2 


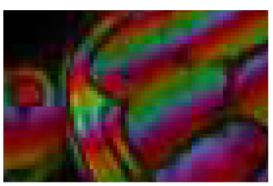

(a) Out of phase image

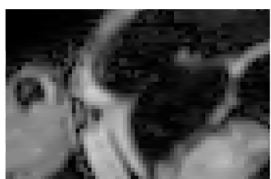

(b) Fat image (Ma method)

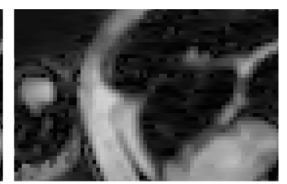

(c) Fat image (our method)

Fig. 3. Close-up of a region in data set 2 to highlight the difference between the methods that occur in some regions

region growing method (visible as a darker area in this image) while our method has classified the region correctly. The resulting images have been qualitatively evaluated with good results. Extensive quantitative evaluation using phantoms will be performed in the near future.

\section{Discussion}

The experiments show that the two methods generate similar results, although there are regions in the data where differences occur. This is in some cases a result of parameter settings. The parameters for the region growing method were initially set equal to the ones used in [1]. These were found not to be optimal and were therefore changed to generate better results. Despite this, misclassifications such as that shown in the second example above occurs. It is possible to correct this by changing the parameters. This, however, results in errors in other slices and data sets. Since the proposed method estimates the phase iteratively, exact parameter selection is less crucial than for the region growing method. This makes the proposed method well suited for fully automatic processing of large data sets such as that acquired in the fat accumulation study.

In some slices, particularly in the presence of large air-filled cavities between anatomic structures, two-dimensional methods (both ours and the region growing method) may both fail, interchanging fat and water. An advantage of the presented method is that it is trivially extensible to three-dimensional correction, while this is rather difficult with region growing-based approaches. Preliminary results indicate that three-dimensional correction using the presented method alleviates the problems near air cavities.

The computational cost of the presented method is slightly higher than that of the region growing. Processing one two-dimensional image using our method takes approximately 5 seconds on a $2.4 \mathrm{GHz} \mathrm{PC}$ while our implementation of the region growing method takes approximately 3 seconds.

\section{References}

1. Ma, J.: Breath-hold water and fat imaging using a dual-echo two-point dixon technique with an efficient and robust phase-correction algorithm. Magnetic Resonance in Medicine 52(2), 415-419 (2004) 
2. Fishbein, M., Mogren, C., Gleason, T., Stevens, W.: Relationship of hepatic steatosis to adipose tissue distribution in pediatric nonalcoholic fatty liver disease. J. Pediatr. Gastroenterol. Nutr. 42(1), 83-88 (2006)

3. Liou, T.H., Chan, W., Pan, L., Lin, P., Chou, P., Chen, C.: Fully automated largescale assessment of visceral and subcutaneous abdominal adipose tissue by magnetic resonance imaging. International Journal of Obesity 30(5), 844-852 (2006)

4. Donnelly, L., O'Brien, K., Dardzinski, B.: Using a phantom to compare MR techniques for determining the ratio of intraabdominal to subcutaneous adipose tissue. American journal of roentgenology 180(4), 993-998 (2003)

5. Dixon, W.: Simple proton spectroscopic imaging. Radiology 153(1), 189-194 (1984)

6. Szumowski, J., Coshow, W., Li, F., Quinn, S.: Phase unwrapping in the three-point dixon method for fat suppression MR imaging. Radiology 192, 555-561 (1994)

7. Akkerman, E., Maas, M.: A region-growing algorithm to simultaneously remove dephasing influences and separate fat and water in two-point dixon imaging. In: Proceedings of the ISMRM Annual Meeting, Nice, France, ISMRM, p. 649 (1995)

8. Song, S.M.H., Napel, S., Pelc, N.J., Glover, G.H.: Phase unwrapping of MR phase images using Poisson equation. IEEE Transaction on Image Processing 4(5), 667676 (1995)

9. Knutsson, H., Westin, C.F.: Normalized and differential convolution: Methods for interpolation and filtering of incomplete and uncertain data. In: Proceedings of IEEE Computer Society Conference on Computer Vision and Pattern Recognition, pp. 515-523 (1993)

10. Horn, B.K.P., Brooks, M.J.: The variational approach to shape from shading. Computer Vision, Graphics, and Image Processing 33, 174-208 (1986)

11. Press, W.H., Flannery, B.P., Teukolsky, S.A., Vetterling, W.T.: Numerical Recipes. Cambridge University Press, Cambridge (1986)

12. Wesseling, P.: An Introduction to Multigrid Methods. Wiley \& Sons, New York (1992) 